\title{
Characterizing spontaneous induction of Stx encoding phages using a selectable reporter system
}

\author{
Jonathan Livny ${ }^{1}$ and David I. Friedman ${ }^{1,2}$ \\ ${ }^{1}$ Program in Cell and Molecular Biology, University of \\ Michigan, Ann Arbor, MI 48109, USA. \\ ${ }^{2}$ Department of Microbiology and Immunology, University \\ of Michigan, Ann Arbor, MI 48109, USA.
}

\section{Summary}

Shiga toxin (Stx) genes in Stx producing Escherichia coli (STEC) are encoded in prophages of the $\lambda$ family, such as H-19B. The subpopulation of STEC lysogens with induced prophages has been postulated to contribute significantly to Stx production and release. To study induced STEC, we developed a selectable in vivo expression technology, SIVET, a reporter system adapted from the RIVET system. The SIVET lysogen has a defective $\mathrm{H}-19 \mathrm{~B}$ prophage encoding the TnpR resolvase gene downstream of the phage $P_{\mathrm{R}}$ promoter and a cat gene with an inserted tet gene flanked by targets for the TnpR resolvase. Expression of resolvase results in excision of tet, restoring a functional cat gene; induced lysogens survive and are chloramphenicol resistant. Using SIVET we show that: (i) approximately $0.005 \%$ of the $\mathrm{H}-19 \mathrm{~B}$ lysogens are spontaneously induced per generation during growth in LB. (ii) Variations in cellular physiology (e.g. RecA protein) rather than in levels of expressed repressor explain why members of a lysogen population are spontaneously induced. (iii) A greater fraction of lysogens with stx encoding prophages are induced compared to lysogens with non-Stx encoding prophages, suggesting increased sensitivity to inducing signal(s) has been selected in Stx encoding prophages. (iv) Only a small fraction of the lysogens in a culture spontaneously induce and when the lysogen carries two lambdoid prophages with different repressor/operators, 933W and H-19B, usually both prophages in the same cell are induced.

\section{Introduction}

Bacterial virulence factors are often encoded within the genomes of prophages (Barksdale and Arden, 1974;

Accepted 10 November, 2003. *For correspondence. E-mail davidfri@umich.edu; Tel. (+1) 734763 3142; Fax (+1) 7347643562.
Boyd and Brussow, 2002; Wagner et al., 2002). Induction of toxin encoding prophages has been shown to markedly increase toxin production by a number of bacterial pathogens, including Corynebacterium diphtheria, Vibrio cholerae, Streptococcus pyogenes, and Shiga toxin producing Escherichia coli (STEC) (Wagner and Waldor, 2002), suggesting that prophage induction can play a direct and significant role in modulating the expression of phageencoded virulence factors.

In nearly all strains of STEC isolated to date, the genes encoding the two subunits of Shiga toxin (Stx) are located in the genomes of resident prophages belonging to the lambdoid family of bacteriophages (Newland and Neill, 1988; Datz et al., 1996; Unkmeir and Schmidt, 2000). Although STEC strains can express a number of factors that contribute to virulence, their ability to express Stx has been implicated in the most severe systemic manifestations associated with STEC infections (Sjogren et al., 1994; Heyderman et al., 2001). These systemic sequelae, which include haemorrhagic colitis, haemolytic uremic syndrome, and neurological disorders, can progress to be life-threatening, particularly in young children and the elderly (Karch et al., 1999).

The lambdoid phage $\mathrm{H}-19 \mathrm{~B}$, obtained from the clinically isolated serogroup O26 E. coli $\mathrm{H} 19$, encodes Stx1, one of the two major variants of Stx (Smith and Linggood, 1971; O'Brien et al., 1984; Calderwood et al., 1987; De Grandis et al., 1987). The repressed prophage is integrated in the bacterial chromosome and is replicated as part of the host genome. Our understanding of the nature of lysogeny by lambdoid phages is based primarily on the detailed information gained from studies with $\lambda$ (Hendrix et al., 1983). The prophage state can be maintained as long as $\mathrm{Cl}$ repressor levels are sufficient (Ptashne, 1992). Cl is inactivated by an auto-catalytic cleavage reaction that at high $\mathrm{pH}$ in vitro can occur efficiently in the absence of other proteins, but that at physiological $\mathrm{pH}$ is stimulated by activated RecA (Roberts and Devoret, 1983; Little, 1984; Kim and Little, 1993). RecA activation is mediated by single-stranded DNA as part of the bacterial SOS response following DNA damage (Little and Mount, 1982; Friedberg et al., 1995). Hence, exposure of lysogens to agents that cause DNA damage, such as mitomycin $\mathrm{C}$, results in a sharp decrease in the cellular concentration of functional $\mathrm{Cl}$ repressor (Shinagawa and Ito, 1973). If Cl levels are sufficiently reduced, 
phage lytic growth ensues. This transition from the repressed lysogenic state to the expression of phage lytic functions is known as induction. Lytic growth is characterized by autonomous phage genome replication, production of progeny viral particles, and phage mediated host cell lysis (Hendrix et al., 1983). Thus both host-cell death and release of free phage particles can serve as indicators of prophage induction.

Lysogens with Stx encoding prophages in culture produce free phage in the absence of an inducing agent, suggesting that some prophages undergo spontaneous induction in vitro. The fraction of $\mathrm{H}-19 \mathrm{~B}$ lysogens that spontaneously liberate phage appears to be relatively low, as the amount of phage obtained is orders of magnitude less than the phage titre produced when the same number of lysogens is treated with an inducing agent. These findings indicate that even grown in the absence of known inducing stimuli, the lysogens are comprised of two populations, one in which the prophage is repressed and a significantly smaller one in which the prophage has been induced. The release of phage from a lysogen in the absence of a known inducing agent has been called 'spontaneous phage release' (Lwoff, 1953). We will refer to that event as well as to prophage induction that results in host cell lethality without proof of viable phage production as 'spontaneous induction'. Moreover, we will refer to any loss of prophage repression that, in the absence of a known inducing agent, leads to expression of a phage encoded product, even if it does not result in phage production or host lethality, as 'spontaneous derepression'.

Studies on the regulation of gene expression from the $\mathrm{H}-19 \mathrm{~B}$ genome led to the suggestion that the subpopulation of induced STEC lysogens plays a major role in the production and release of Stx1 (Neely and Friedman, 1998a; Wagner et al., 2002). Beyond the early classic experiments using the micromanipulator to look at the fate of a single bacterium (Lwoff, 1953), little, besides the fact that there is a small yield of phage particles, is known about the fraction of the lysogen population that is spontaneously induced. Even less is known about the fraction of lysogens that is induced during STEC infection of a mammalian host. Furthermore, the factors that cause a lysogen to be induced either spontaneously in vitro or in vivo, in the animal, are poorly understood. Studying the induced lysogen population presents a significant challenge, requiring a method to isolate and study bacteria that are normally destroyed by the phage lytic functions and likely comprise a small fraction of the total population of lysogens. To this end we have constructed a reporter lysogen that survives induction and, further, upon derepression of transcription from the early phage promoter $P_{\mathrm{R}}$, undergoes a heritable and selectable genotypic change, thus allowing for the easy identification of the progeny of induced lysogens even when they represent a small proportion of the total lysogen population. This approach, combining adaptations of the recombinasebased in vivo expression technology (RIVET) described by Camilli et al. (1994) and the $\lambda$ prophage developed for reporting DNA damage by Toman et al. (1985), provides a method for directly isolating and quantifying cells in which the prophage has been induced. In addition, the selectable RIVET system, called SIVET, extends the utility of the RIVET system by facilitating the positive selection for bacteria in which expression from a promoter of choice has been turned on. The SIVET approach was used to measure the fraction of $\mathrm{H}-19 \mathrm{~B}$ lysogens that is induced under standard culture conditions, compare the inducibility of $\mathrm{H}-19 \mathrm{~B}$ and a number of other lambdoid prophages, and examine the factors within the bacterial cell that cause a lysogen to undergo spontaneous induction.

\section{Results}

Constructing a reporter $\mathrm{H}-19 \mathrm{~B}$ lysogen that undergoes a heritable, phenotypic change upon prophage derepression

The recombinase based in vivo expression technology (RIVET) (Camilli et al., 1994; Camilli and Mekalanos, 1995) was developed as a method for identifying the progeny of bacterial cells in which a promoter of interest has been activated. The main limitation of this approach is that the progeny of cells in which expression had been activated are isolated based on their sensitivity to tetracycline; isolating cells by screening or even by selection with fusaric acid (Bochner et al., 1980) becomes impractical when the targeted cells represent an extremely small proportion of the total population. To circumvent this limitation, we developed a reporter system adapted from RIVET, known as selectable in vivo expression technology (SIVET), in which activation of transcription from a promoter of interest results in the acquisition of a selectable and heritable gain of chloramphenicol resistance (Fig. 1). The SIVET system is comprised of two components. The first is the gene encoding the $\gamma \delta \mathrm{TnpR}$ resolvase (Reed et al., 1982; Grindley, 1983; Heffron, 1983) inserted downstream of a promoter of interest. The second is a chloramphenical transacetylation gene (cat) disrupted by an inserted tetracycline resistance gene (tet) flanked by altered resolvase target sequences (resC). Upon expression of $\mathrm{TnpR}$, the tet gene and one resC site are excised, leaving one res $C$ site inserted in cat. Excision results in a gene encoding a functional Cat protein, rendering the cell and its progeny resistant to chloramphenicol. Modification of res sites to res $C$ was necessary because the res sequence remaining in the cat gene following excision would introduce a TAG stop codon in the cat reading frame. To eliminate this stop codon, the guanine of the 


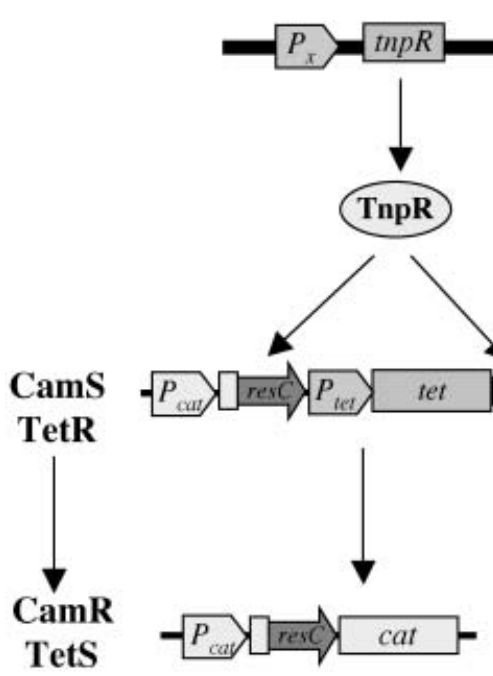

TetS

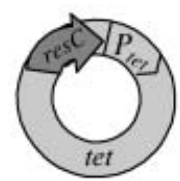

Fig. 1. General scheme of the selectable in vivo expression technology (SIVET). Expression of TnpR following activation of transcription from a promoter of interest results in excision and circularization of the tet gene and one modified resolvase excision site (resC). The excised DNA does not replicate and thus is lost by segregation. Excision of tet creates a functional resC-cat ORF. TnpR does not catalyse the reinsertion of tet, which is lost through segregation. Thus cells in which TnpR has been expressed undergo a genetic conversion which renders them and their progeny resistant to chlorampheni$\mathrm{col}$ and sensitive to tetracycline. Not drawn to scale.

TAG codon was changed to a cytosine in both res sites flanking the tet gene, producing res $C$ sites.

To apply the SIVET scheme to identification and isolation of derepressed lysogens, we constructed a reporter $\mathrm{H}-19 \mathrm{~B}$ prophage in which TnpR expression is dependent on transcription from the early phage promoter $P_{\mathrm{R}}$. The tnpR gene with its ribosomal binding site (RBS) was inserted into the $\mathrm{H}-19 \mathrm{~B}$ prophage downstream of $P_{\mathrm{R}}$ (Fig. 2). As first shown in $\lambda$ and much later in H-19B, transcription from $P_{\mathrm{R}}$ is regulated by $\mathrm{Cl}$ repressor, which inhibits transcription by binding at both the $P_{\mathrm{R}}$ associated $O_{\mathrm{R}}$ and $P_{\mathrm{L}}$ associated $O_{\mathrm{L}}$ operators (Gussin et al., 1983; Ptashne, 1992; Shi and Friedman, 2001). To ensure that induction of the TnpR expressing prophage closely approximates the normal process of prophage induction, tnp $R$ was inserted into the $\mathrm{H}-19 \mathrm{~B}$ genome downstream of the cro gene, a location that would prevent the inserted gene from interfering with expression of phage functions and/or activity of sites involved in repression and derepression of the prophage. Although repressor binding at $O_{\mathrm{L}}$ does not directly influence transcription initiating at $P_{\mathrm{R}}$, the design of the reporter prophage included an intact $O_{L}$. This design feature was based on findings that, in the wildtype $\lambda$ prophage, repressor bound to $O_{R}$ is further stabilized by its interaction with repressor bound at $O_{\mathrm{L}}$ (Revet et al., 1999; Dodd et al., 2001).

Following induction, lambdoid prophages express a number of functions that lead to the death of the bacterial host cell (Court and Oppenheim, 1983). Therefore, the tnpR encoding prophage was modified to allow bacterial survival following induction. To this end we adopted a strategy based on the findings that host cell death following induction of a $\lambda$ lysogen could be prevented by the inactivation of two phage genes (Eisen et al., 1968): $N$, encoding a transcription elongation protein, and either $O$ or $P$, encoding proteins required for initiation of phage DNA replication. We replaced the $\mathrm{H}-19 \mathrm{~B} N$ gene with the kanamycin resistance gene (kan) and the adjacent $O$ and $P$ genes, with the ampicillin resistance gene $(\mathrm{amp})$ yielding the H-19B $\Delta N$ ::kan $\triangle O P:: a m p$ prophage (Fig. 2). Following exposure to mitomycin $\mathrm{C}$, the number of surviving $\mathrm{H}-19 \mathrm{~B} \Delta \mathrm{N}$ ::kan $\triangle O P$ ::amp lysogens was over four logs greater compared to the number of surviving wild-type $\mathrm{H}$ $19 \mathrm{~B}$ lysogens and similar to the number of surviving nonlysogenic MC1000 cells. The tnpR gene was recombined into the $\triangle N$ ::kan $\triangle O P$ ::amp downstream of $P_{\mathrm{R}}$. This prophage, H-19B SIVET, was introduced into a derivative of strain MC1000 in which the galK gene had been replaced by the resC-tet-res $C$ disrupted cat gene.

The following summarizes how the components of SIVET function to identify lysogens in which derepression of the $\mathrm{H}-19 \mathrm{~B}$ prophage has taken place (Figs 1 and 2). Repressor inactivation leads to transcription from the $P_{\mathrm{R}}$ promoter resulting in expression of TnpR. TnpR excises
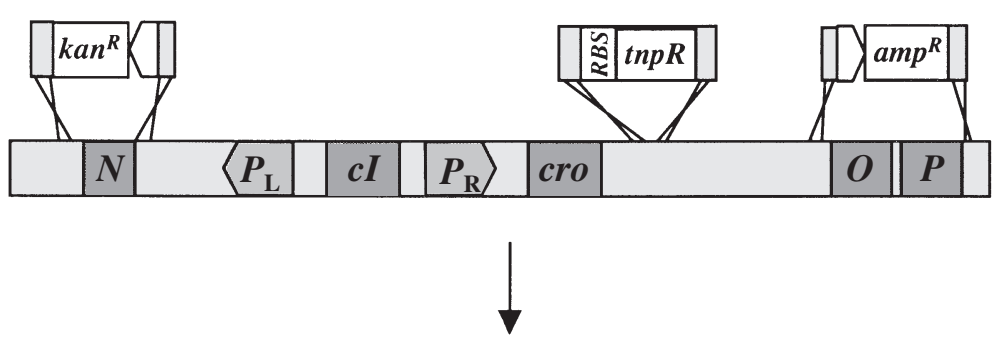

Fig. 2. Regulatory region of $\mathrm{H}-19 \mathrm{~B}$ showing relevant genes and sites of allele replacement. Host killing by induced prophage was eliminated by replacing the $N$ gene with a kanamycin resistance gene (kan) and the $O$ and $P$ genes with an ampicillin resistance gene (amp). The tnp $R$ resolvase gene with its RBS was introduced into the $\mathrm{H}$-19B prophage downstream of the cro gene in the $P_{\mathrm{R}}$ operon. Not drawn to scale.

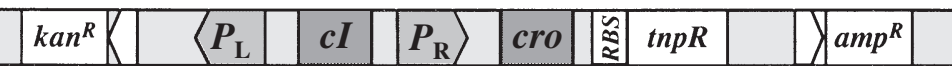

(C) 2004 Blackwell Publishing Ltd, Molecular Microbiology, 51, 1691-1704 
the res $C$-tet-res $C$ disrupting the cat gene in the chromosome, changing the phenotype of the lysogen from TetR to CamR. Thus, the SIVET scheme renders the progeny of cells that have expressed TnpR easily identifiable by selection for CamR. Because the SIVET prophage encodes resistance to kanamycin and ampicillin and cannot excise following induction, all SIVET lysogens, induced and non-induced, are resistant to those antibitotics. Thus the fraction of induced lysogens can be determined by dividing the number of cells resistant to chloramphenicol by the number of cells resistant to kanamycin and/or ampicillin.

\section{Comparing the acquisition of CamR in the SIVET lysogen to wild-type prophage induction}

The SIVET lysogen was used to measure resC-tet-resC excision under standard culture conditions. To minimize the background of lysogens in which resC-tet-res $C$ had been excised, the SIVET lysogens were grown in LB supplemented with tetracycline before being diluted in LB and grown to stationary phase. Following growth to stationary phase in LB, approximately $0.11 \%$ of SIVET lysogens became CamR (Fig. 3). We note that as acquisition of CamR by the reporter lysogens is heritable and irreversible, the CamR SIVET lysogens isolated represent the progeny of all cells that are induced throughout growth of the culture. The SIVET lysogens underwent approxi-

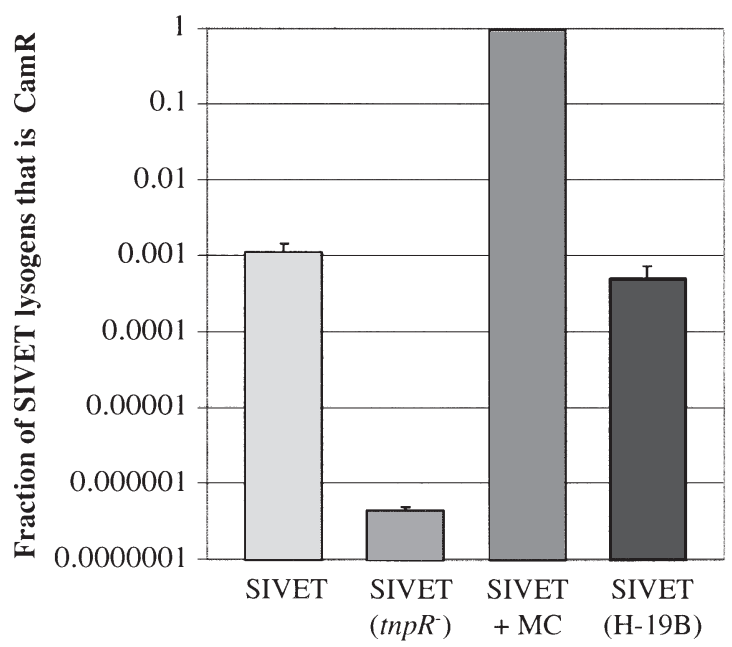

Fig. 3. Effect of TnpR expression from the H-19B SIVET prophage on the frequency of obtaining CamR lysogens. Cultures were grown to stationary phase in LB broth, some following treatment with mitomycin $C(M C)$, and titres of induced and uniduced lysogens were determined using appropriate selection. See Experimental procedures for details. SIVET (tnp $R^{-}$) does not encode the tnpR gene; SIVET(H-19B) also carries a wild-type $\mathrm{H}-19 \mathrm{~B}$ prophage. Fraction that are CamR is calculated as the number of colonies resistant to Cam, Amp, and Kan/the number of colonies resistant to Amp and Kan. Values shown correspond to the mean of at least three independent experiments with error bars indicating the standard error. mately six rounds of cell division in the absence of antibiotics before reaching stationary phase. Assuming a similar fraction of the total population was spontaneously induced in each generation, the fraction of lysogens spontaneously induced per generation is approximately one-seventh of the fraction of lysogens that are CamR following growth to stationary phase, i.e. $0.016 \%$ or 1 in 6400 lysogens acquired CamR every generation. Acquisition of CamR is dependent on expression of TnpR, decreasing by three orders of magnitude in a SIVET strain lacking tnpR. Exposure of the SIVET lysogen to mitomycin C resulted in $95 \%$ of SIVET lysogens becoming CamR, suggesting that, like the wild-type $\mathrm{H}-19 \mathrm{~B}$ lysogen, derepression leading to transcription from $P_{\mathrm{R}}$ promoter in the SIVET lysogen was stimulated by activation of the host SOS response. Additional proof for this assertion will be provided below.

Studies with RIVET have shown that extremely low levels of TnpR expression can result in excision of restet-res rendering the assay so sensitive as to interfere with its effectiveness at identifying active promoters (Lee et al., 1999). This prompted concern that very low levels of transcription from $P_{\mathrm{R}}$, although not sufficient to result in cell death through lytic development of a wild-type prophage, might result in sufficient expression of TnpR to excise resC-tet-res $C$. To determine how closely the level of derepression needed to excise resC-tet-res $C$ in the SIVET lysogen corresponds to that required for induction of wild-type $\mathrm{H}-19 \mathrm{~B}$, a SIVET lysogen carrying a wild-type $\mathrm{H}-19 \mathrm{~B}$ prophage, SIVET(H19B), was constructed and used to determine the number of lysogens in which prophage derepression leads to excision of resC-tet-resC but not killing by the wild-type prophage. The reasoning behind this strategy anticipates two possible outcomes. If the level of derepression that is sufficient to excise res $C$ tet-resC from the SIVET(H19B) lysogen is the same as the level required to activate the wild-type prophage, in nearly every bacterium in which derepression results in excision of resC-tet-res $C$, the wild-type prophage would be induced, resulting in death of the lysogen. Thus, the number of CamR colonies would be very low. Alternatively, if the level of derepression causing excision of resC-tet-resC in the SIVET(H19B) is significantly lower than that required to activate the wild-type prophage, in many bacteria in which the resC-tet-res $C$ is excised, the wild-type prophage would not be induced. Thus the lysogen would survive and there would be nearly as many CamR colonies as found with the SIVET lysogen control.

The fraction of SIVET(H19B) lysogens that were CamR was only slightly lower than the fraction of SIVET lysogens that were CamR (Fig. 3) suggesting that, in a significant proportion of SIVET lysogens that lose resCtet-res $C$, the wild-type $\mathrm{H}-19 \mathrm{~B}$ prophage does not express 
sufficient levels of phage functions to kill the host cell. Thus the low amounts of TnpR expression sufficient to cause resC-tet-res $C$ excision make the SIVET lysogen, as constructed, too sensitive to provide an accurate measurement of the absolute number of wild-type $\mathrm{H}-19 \mathrm{~B}$ lysogens that have undergone spontaneous induction. Furthermore, this result demonstrates that, in a subset of lysogens, derepression is sufficient to allow some transcription from $P_{\mathrm{R}}$ but not sufficient to result in levels of phage lytic function expression necessary to threaten cell viability.

We refer to the general approach of using a SIVET lysogen with a second intact prophage to compare the inducibility of the SIVET prophage with the inducibility of the intact prophage as the SIVET induction index test (IIT). The inducibility index refers to the ratio of the CamR fraction of SIVET lysogens with the intact second prophage to the CamR fraction of SIVET lysogens. The lower the ratio, the more similar is the inducibility of the intact prophage to the inducibility of the SIVET prophage. When the ratio is extremely low, it is not possible to discern whether the two phages induce similarly or the intact phage induces more readily.

\section{Optimizing SIVET sensitivity}

We adopted the strategy used to decrease the sensitivity of the RIVET procedure by Lee et al. (1999) to modify the SIVET lysogen so the fraction that are CamR closely reflects the fraction of spontaneously induced lysogens with a wild-type $\mathrm{H}-19 \mathrm{~B}$ prophage. The ribosome bindings site (RBS) of tnpR was altered to increase the amount of tnpR transcription needed to attain levels of TnpR suffi- cient for excision of resC-tet-resC. Two derivatives of the SIVET lysogen were constructed, each carrying one of the mutations in the RBS of tnpR reported by Lee et al. (1999), tnpR168 and tnpR184, yielding strains SIVET ${ }^{168}$ and SIVET ${ }^{184}$. When cultures of the lysogens were grown to stationary phase in LB, $0.03 \%$ and $0.01 \%$ of SIVET ${ }^{168}$ and SIVET $^{184}$ lysogens, respectively, acquired CamR (Fig. 4). This corresponds to approximately $0.0043 \%$ and $0.0014 \%$ of SIVET $^{168}$ and SIVET ${ }^{184}$ cells, respectively, acquiring CamR per generation. When the two derivative strains were exposed to mitomycin C, $96 \%$ of SIVET ${ }^{168}$ cells and only $8 \%$ of SIVET ${ }^{184}$ cells became CamR (Fig. 4).

We next employed SIVET IIT to determine how closely resC-tet-resC excision in SIVET ${ }^{168}$ and SIVET ${ }^{184}$ corresponds to wild-type $\mathrm{H}-19 \mathrm{~B}$ prophage induction. For both RBS-mutant derivatives carrying the wild-type $\mathrm{H}-19 \mathrm{~B}$ prophage, very few CamR lysogens were isolated compared to the strains carrying only the SIVET prophage (Fig. 4). These results suggest that, unlike the SIVET ${ }^{\mathrm{WT}}$ $(\mathrm{H} 19 \mathrm{~B})$ strain, in nearly every $\operatorname{SIVET}^{168}(\mathrm{H} 19 \mathrm{~B})$ and $\operatorname{SIVET}^{184}(\mathrm{H} 19 \mathrm{~B})$ lysogen in which resC-tet-resC was excised, the wild-type prophage was induced. Thus, the fraction of SIVET ${ }^{168}$ and SIVET ${ }^{184}$ lysogens that become CamR is equal to or less than the fraction of lysogens that are spontaneously induced as measured by lethality to the host bacterium. Because SIVET ${ }^{168}$ was found to spontaneously yield a higher fraction of CamR lysogens than did SIVET $^{184}$ yet was shown by the SIVET IIT assay not to be overly sensitive to low levels of transcription from $P_{\mathrm{R}}$, SIVET ${ }^{168}$ is the derivative in which acquisition of CamR most closely reflects spontaneous induction of the wildtype $\mathrm{H}-19 \mathrm{~B}$ lysogen.

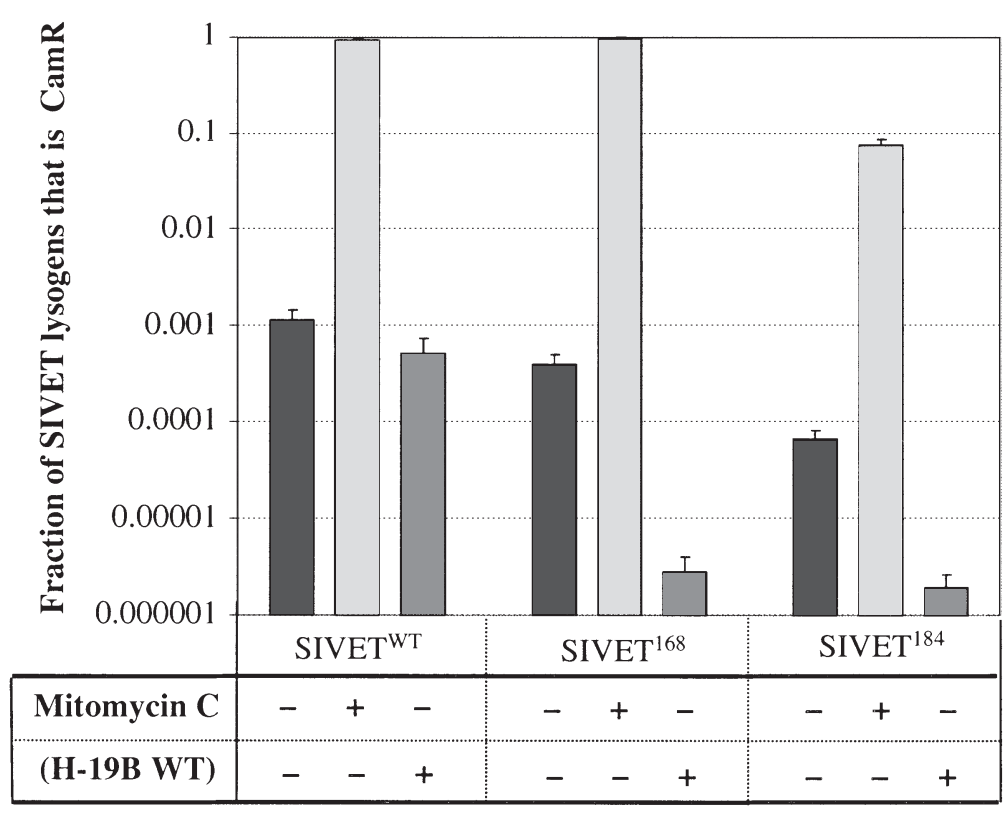

Fig. 4. Fraction of SIVET lysogens that is CamR in cultures of SIVET lysogens altered in their ability to express TnpR. Strains listed as SIVET $^{168}$ (K10449) and SIVET ${ }^{184}$ (K10450) have nucleotide changes at different positions in the tnpR-associated RBS. Cultures were grown to stationary phase in LB broth, some following treatment with mitomycin $\mathrm{C}$ and titres of induced and uninduced lysogens were determined using appropriate selection. See Experimental procedures for details. Fraction that are CamR is calculated as described in Fig. 3. Values shown correspond to the mean of at least three independent experiments with error bars indicating the standard error. 
Assessing the role of $\mathrm{Cl}$ auto-cleavage in $\mathrm{H}-19 \mathrm{~B}$ spontaneous induction

To confirm that spontaneous $\mathrm{H}-19 \mathrm{~B}$ induction required RecA mediated $\mathrm{Cl}$ autocleavage, we introduced a mutation into the $\mathrm{cl}$ gene of the SIVET prophage predicted to abolish auto-cleavage of the $\mathrm{Cl}$ protein (J.W. Little, pers. comm.). This prediction was based on work with other cleavable repressors, in which mutations changing either a conserved serine or lysine residue were shown to abrogate auto-catalytic activity (Lin and Little, 1988). Based on its location a likely candidate for the conserved serine codon in the $c$ l gene of the prophage in SIVET ${ }^{168}$ was replaced with an alanine codon, creating the clind mutant allele. When the $\mathrm{H}-19 \mathrm{~B} \mathrm{SIVET}^{168}$ prophage encodes the clind allele, the fraction of lysogens that spontaneously acquire CamR is decreased by three logs compared to SIVET $^{168}$ (Fig. 5). This approaches the number of CamR lysogens observed in cultures of the SIVET derivative missing tnpR. These findings confirm that spontaneous induction of the SIVET lysogen, as expected, is due to host mediated $\mathrm{Cl}$ degradation. A culture of SIVET ${ }^{\mathrm{WT}}$ lysogens with a clind SIVET prophage also spontaneously yielded extremely small numbers of CamR colonies similar to the numbers yielded by the SIVET lysogen missing a functional tnpR gene (Fig. 5). Hence, even low levels

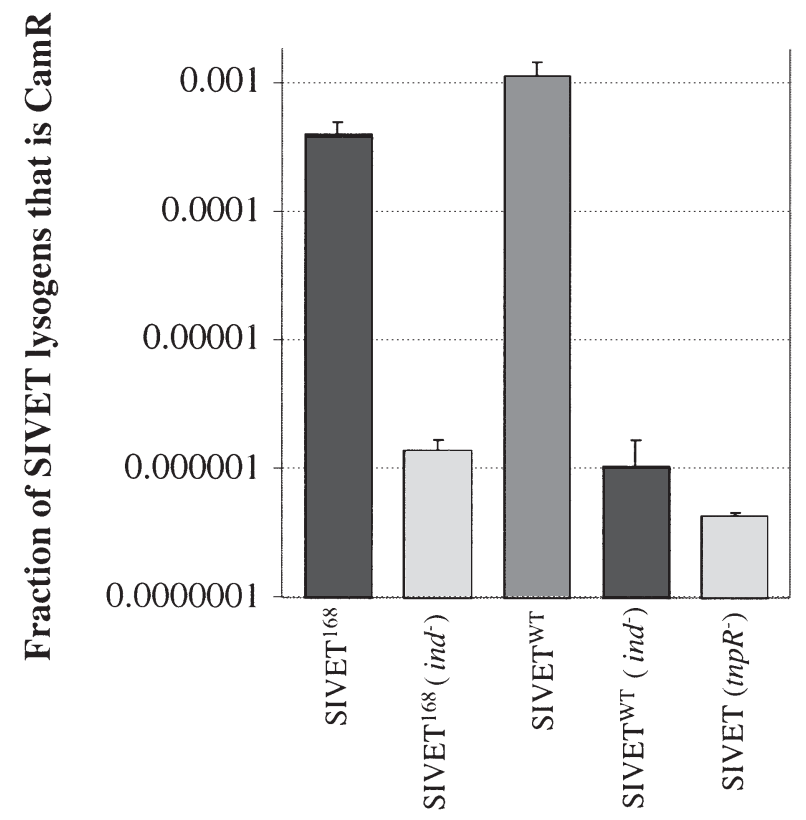

Fig. 5. Role of autocleavage in spontaneous induction of SIVET lysogens. Cultures were grown to stationary phase in LB broth and titers determined using appropriate selection. See Experimental procedures for details. Changes in the SIVET lysogens are indicated in parenthesis: ind, a mutation in the $c$ gene resulting in a repressor that does not autocleave; $t n p R^{-}$, no resolvase gene. Fraction of lysogens that is CamR was determined as described for Fig. 3. Values shown correspond to the mean of at least three independent experiments with error bars indicating the standard error. of transcription from $P_{\mathrm{R}}$ that fail to promote prophage induction require repressor inactivation.

Determining the fraction of lysogens carrying the Stx2 encoding phage $933 \mathrm{~W}$ that undergo spontaneous induction

We next applied the SIVET system to identify and quantify the fraction of lysogens carrying the Stx encoding lambdoid phage $933 \mathrm{~W}$ that are induced. $933 \mathrm{~W}$ carries the genes encoding Stx2, the other major variant of Stx (O'Brien et al., 1984; Plunkett et al., 1999). Whereas 933 $\mathrm{W}$ and $\mathrm{H}-19 \mathrm{~B}$ share similar genomic organizations as well as some genes (Neely and Friedman, 1998a,b; Plunkett et al., 1999), they have different immunities, meaning that each phage expresses a distinct $\mathrm{Cl}$ that cannot mediate repression of the other (O'Brien et al., 1984). A SIVET ${ }^{168}$ lysogen in which the SIVET prophage has the immunity of $933 \mathrm{~W}$ rather than the immunity of $\mathrm{H}-19 \mathrm{~B}$ was constructed and named $933 \mathrm{~W}$ SIVET ${ }^{168}$. We retain the nomenclature of SIVET without indication of the immunity for all constructs with SIVET prophages having the immunity of $\mathrm{H}-19 \mathrm{~B}$. Approximately $0.10 \%$ of the 933 W SIVET ${ }^{168}$ lysogens acquired Cam resistance spontaneously when grown to stationary phase in LB. Correcting for the accumulation of CamR lysogens from previous rounds of cell division, this corresponds to approximately $0.014 \%$ or 1 in 7000 per cell generation.

\section{Comparing the inducibility of $\mathrm{H}-19 B$ with that of other lambdoid phages}

The SIVET IIT approach was used to compare the inducibility of the SIVET ${ }^{168} \mathrm{H}$-19B prophage with the inducibility of lambdoid prophages encoding other immunity regions. The SIVET ${ }^{168}$ strain was lysogenized with $933 \mathrm{~W}, \lambda, \mathrm{P} 21$, or $\lambda i m m 434$, yielding strains $\operatorname{SIVET}^{168}(933 \mathrm{~W})$, $\operatorname{SIVET}^{168}(\lambda), \operatorname{SIVET}^{168}(\mathrm{P} 21)$, and $\operatorname{SIVET}^{168}(\lambda i m m 434)$. Following growth of these double lysogen strains to stationary phase, the fraction of $\operatorname{SIVET}^{168}(933 \mathrm{~W})$ cells that was CamR was found to be nearly 30 -fold less than that observed in the SIVET ${ }^{168}$ strain (Fig. 6), indicating that in almost every cell in which the $\mathrm{H}-19 \mathrm{~B}$ prophage was induced the $933 \mathrm{~W}$ prophage was also induced. The number of CamR SIVET ${ }^{168}(\lambda i m m 434)$ lysogens obtained was reduced only about threefold compared to SIVET ${ }^{168}$, indicating that in a significant subset of cells in which SIVET ${ }^{168}$ had been induced, the $\lambda$ imm434 prophage had not been induced (Fig.6). This threefold decrease was substantially less than that seen in $\operatorname{SIVET}^{168}(933 \mathrm{~W})$, suggesting that the fraction of lysogens with a $\lambda i m m 434$ prophage undergoing spontaneous induction is significantly less than lysogens with either $\mathrm{H}-19 \mathrm{~B}$ or $933 \mathrm{~W}$ prophages. The fractions of $\operatorname{SIVET}^{168}(\lambda)$ and 


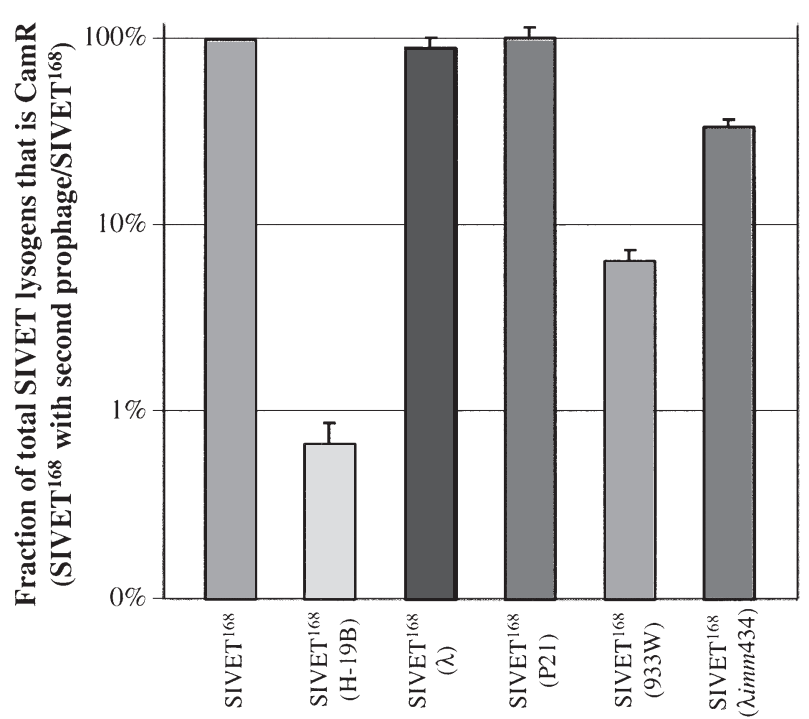

Fig. 6. Comparison of spontaneous induction frequencies of $\mathrm{H}-19 \mathrm{~B}$ and lambdoid phages with different repressor-operator systems (immunity regions) using SIVET IIT. Cultures were grown to stationary phase in LB broth and titers determined using appropriate selection. See Experimental procedures for details. Indicated in parenthesis are the intact second prophages. The indicated values are normalized to the fraction of total SIVET ${ }^{168}$ lysogens that are CamR which is set at $100 \%$ and represented by the left most bar. Raw data were calculated as the number of colonies resistant to Cam, Amp, and Kan/the number of colonies resistant to Amp and Kan. Values shown correspond to the mean of at least three independent experiments with error bars indicating the standard error.

SIVET $^{168}(\mathrm{P} 21)$ that were CamR were similar to those observed in SIVET ${ }^{168}$ (Fig. 6), indicating that, in the vast majority of cells in which resC-tet-res $C$ is excised, neither $\lambda$ nor P21 were induced. These findings suggest that spontaneous induction of $\mathrm{H}-19 \mathrm{~B}$ either occurs in a larger fraction of cells than does either $\lambda, \mathrm{P} 21$, or 434 induction, or through a mechanism distinct from that causing induction of $\lambda, P 21$ and 434.

To differentiate between these two possibilities, we repeated SIVET IIT analysis using $\lambda c / 857$ ind $^{+}$or $\lambda \mathrm{O}_{\mathrm{R}} 323$ as the intact prophages. $\lambda \mathrm{cl} 857 \mathrm{ind}^{+}$, a recombinant derived from the original $\lambda c / 857$ ind phage (Sussman and Jacob, 1962), encodes a $c /$ with a point mutation resulting in a relatively unstable repressor (S. Adhya, pers. comm.); $\lambda \mathrm{O}_{\mathrm{R}} 323$ has changes in the right operator (Little et al., 1999). Based on the observation that lysogens with these $\lambda$ prophages spontaneously produce more phage than do wild-type $\lambda$ lysogens (S. Adhya, pers. comm.; Little et al., 1999) we reasoned that these prophages undergo spontaneous induction more readily than wild-type $\lambda$. Thus, if spontaneous induction of $\mathrm{H}-19 \mathrm{~B}$ and $\lambda$ is mediated by the same host-specific factor, SIVET ${ }^{168}$ lysogens carrying these mutant $\lambda$ prophages should produce a lower proportion of CamR cells than SIVET ${ }^{168}$. As shown in Fig. 7, the fraction of lysogens that were CamR was significantly reduced in cultures of the $\operatorname{SIVET}^{168}\left(\lambda c / 857\right.$ ind $\left.^{+}\right)$and $\operatorname{SIVET}^{168}\left(\lambda \mathrm{O}_{\mathrm{R}} 323\right)$ strains. These findings suggest that in nearly every cell in which $\mathrm{H}-19 \mathrm{~B}$ was induced, the $\lambda c / 857$ ind $^{+}$and $\lambda \mathrm{O}_{\mathrm{R}} 323$ prophages were induced as well. Therefore, we conclude that induction of $\mathrm{H}-19 \mathrm{~B}$ is mediated by the same factor, presumably activated RecA, that causes induction of $\lambda$, but that spontaneous $\lambda$ prophage induction requires a relatively higher level of this factor and thus occurs in a smaller proportion of cells than does spontaneous $\mathrm{H}-19 \mathrm{~B}$ induction.

\section{Discussion}

\section{The SIVET system}

Selectable in vivo expression technology was developed to extend the utility of RIVET technology by allowing the isolation of a small fraction of a population of bacteria in which transcription from a promoter of interest had been activated. In the SIVET system, expression of TnpR leads to a genetic change resulting in cells that started as stably TetR and CamS becoming CamR and TetS. Because they are identified by selection rather than by screening, the induced bacteria can be isolated and quantified even when they represent an extremely small fraction of the total population. Furthermore, as acquisition of CamR is

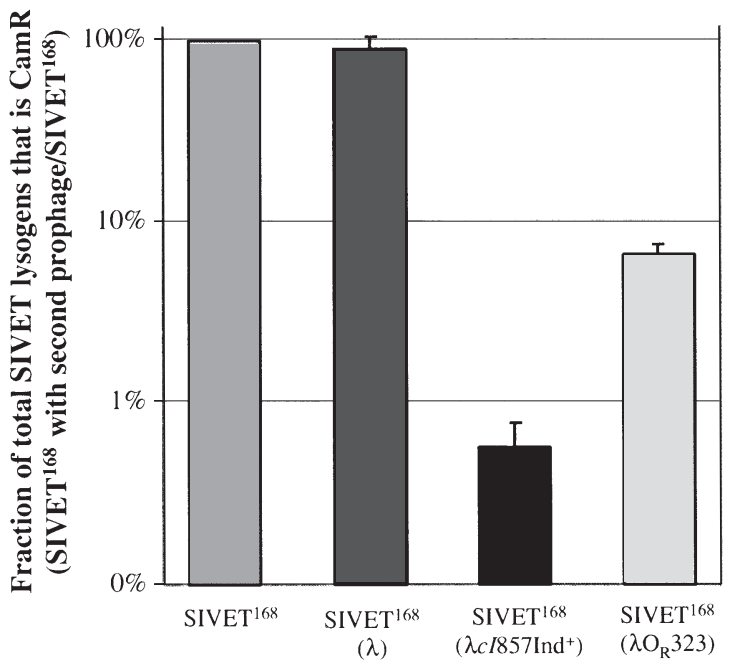

Fig. 7. Comparison of spontaneous induction frequencies of $\mathrm{H}-19 \mathrm{~B}$ and lambdoid phages with repressor-operator systems (immunity regions) containing mutations affecting their inducibility using SIVET IIT. Cultures were grown to stationary phase in LB broth and titers determined using appropriate selection. See Experimental procedures for details. Listed in parenthesis are the intact second prophages; $c / 857 \mathrm{Ind}^{+}$has a point mutation in $\lambda \mathrm{cl}$ and $\lambda \mathrm{O}_{\mathrm{R}} 323$ mutations in the right operator (see text for details). The indicated values are normalized to the fraction of total SIVET ${ }^{168}$ lysogens that are CamR which is set at $100 \%$ and represented by the left most bar. Raw data were calculated as described in Fig. 5. Values shown correspond to the mean of at least three independent experiments with error bars indicating the standard error. 
both irreversible and heritable, identifying induced cells using SIVET does not require that selection take place during growth under the environmental conditions being tested.

The SIVET approach was applied to the study of spontaneous prophage induction in vivo, with a long-term goal of assessing prophage induction during STEC infection. To reduce the sensitivity of the system, we altered the RBS of the tnpR gene as described by Lee et al. (1999). This ability to alter the sensitivity of the system allowed us to make a derivative of the $\mathrm{H}-19 \mathrm{~B}$ SIVET lysogen, SIVET ${ }^{168}$, in which acquisition of CamR closely matched induction of the intact $\mathrm{H}-19 \mathrm{~B}$ prophage. Hence, the fraction of SIVET ${ }^{168}$ lysogens that become CamR per cell generation is a close approximation of the fraction of lysogens carrying the intact $\mathrm{H}-19 \mathrm{~B}$ prophage that undergoes spontaneous induction. We therefore will use induction throughout the rest of the discussion whether discussing either SIVET ${ }^{168}$ or lysogens carrying intact prophages.

\section{Fraction of lysogens spontaneously induced}

Using the SIVET ${ }^{168}$ lysogen, we showed that, under standard culture conditions, approximately 1 in 20000 lysogens carrying the Stx-1 encoding phage $\mathrm{H}-19 \mathrm{~B}$ and 1 in 7000 lysogens carrying the Stx-2 encoding phage 933 $\mathrm{W}$ are spontaneously induced per cell generation. Whereas the fraction of the $\mathrm{H}-19 \mathrm{~B}$ lysogen population that is induced in the intestinal environment was not directly examined here, host derived factors such as pharyngeal cells and neutrophils have been shown to stimulate phage production in vitro by $S$. pyogenes and E. coli lysogens, respectively (Broudy et al., 2001; Wagner et al., 2001a), suggesting that the fraction of the $\mathrm{H}-19 \mathrm{~B}$ and $933 \mathrm{~W}$ lysogen populations induced during infection may be greater than that observed in culture.

\section{On the role of $\operatorname{Rec} A$}

Previous studies with $\lambda$ identified activated RecA as an important contributor to spontaneous induction. First, a lysogen carrying a $\lambda$ ind prophage produces very few phage spontaneously when compared to a lysogen with a $\lambda$ ind $^{+}$prophage (Roberts and Devoret, 1983). Second, a rec $A^{-}$lysogen carrying a $\lambda$ ind $^{+}$prophage shows a similar reduction in spontaneous phage production, indicating that the physiological state of the rec $A^{-}$lysogen results in an Ind $^{-}$phenotype (Brooks and Clark, 1967; Hertman and Luria, 1967). Third, Fuchs et al. (1999) showed that there were unmeasurable levels of phage produced from a rec $A^{-}$derivative of a phage producing EHEC strain.

Our studies with the $\mathrm{H}-19 \mathrm{~B}$ SIVET lysogens address the roles of $\mathrm{Cl}$ expression and inactivation in the sponta- neous induction of the $\mathrm{H}-19 \mathrm{~B}$ prophage. First, the $\mathrm{H}-19 \mathrm{~B}$ SIVET lysogens in which the SIVET prophage $\mathrm{cl}$ gene encodes the ind mutation are essentially uninducible, showing a greater than two log decrease in the fraction of lysogens that becomes CamR. Hence, a host encoded product that stimulates repressor autocleavage, presumably activated RecA, is required for spontaneous induction of $\mathrm{H}-19 \mathrm{~B}$. Second, studies with derivatives of the $\mathrm{H}-19 \mathrm{~B}$ SIVET lysogen carrying an additional prophage provide evidence that variation in levels of repressor expression is likely to play a minor role, if any, in spontaneous derepression leading to prophage induction. When the intact prophage in the SIVET IIT is either $933 \mathrm{~W}$ or $\lambda \mathrm{O}_{\mathrm{R}} 323$, there is a $\sim 95 \%$ decrease in the number of CamR colonies. Hence, in the vast majority of bacteria in which sufficient levels of spontaneous derepression occur, phages expressing different repressor proteins are both induced. Because spontaneous induction of $\mathrm{H}-19 \mathrm{~B}$ and $933 \mathrm{~W}$, as determined by SIVET analysis, is $5 \times 10^{-5}$ and $1.4 \times 10^{-4}$, respectively, if induction of prophages in the same bacterium were independent events then the proportion of cells in which coinduction of $\mathrm{H}-19 \mathrm{~B}$ and $933 \mathrm{~W}$ had occurred would be $\sim 7 \times 10^{-9}$. Therefore, if spontaneous induction of prophages with different repressors occurs independently of each other there should be no observable difference in the number of CamR colonies in the $\mathrm{H}-19 B$ SIVET strain whether or not it carries a $933 \mathrm{~W}$ prophage. As this is obviously not the case, these results provide compelling evidence that stochastic fluctuations of repressor levels within a population of a specific lysogen are unlikely to play a significant role in spontaneous induction. In making this argument we assume that it is unlikely that there is a mechanism dedicated, in some unexplainable way, toward specifically reducing expression of a number of different phage repressor genes in lysogens undergoing spontaneous induction. Therefore, the simplest explanation is that spontaneous induction occurs solely on the basis of the level of activated RecA reaching the critical level for prophage induction.

\section{SIVET as a tool to measure relative inducibility of prophages}

The SIVET lysogen was also used to directly compare levels of spontaneous induction of prophages that differ in their repressors and cognate operators (i.e. differ in their immunity regions). Called the SIVET-induction index test (IIT), the comparison is between the levels of induction of the defective SIVET prophage and a second fully functional prophage with a different immunity region. The prophages tested could be divided into three classes. In one class, composed of phages with the immunity regions of lambdoid phages $\lambda$ and 21 , spontaneous induction occurred in a significantly smaller proportion of 
the lysogen population than did induction of $\mathrm{H}-19 \mathrm{~B}$. In the second class, composed of the Stx encoding phages $933 \mathrm{~W}$, spontaneous induction occurred in a similar fraction of the lysogen population as did induction of H-19B. In the third class, composed of the phage with the immunity of 434 , spontaneous induction occurred in a smaller fraction of lysogens than did induction of $\mathrm{H}-19 \mathrm{~B}$ but in a somewhat higher fraction of lysogens than did induction of prophages with the immunity regions of $\lambda$ or P21. This result is consistent with previous studies showing that phages with the immunity of 434 express less repressor and induce more readily than do phages with the immunity of $\lambda$ (Pirrotta and Ptashne, 1969; Levine et al., 1979).

In this regard it is interesting to note a study by Bertani (1951) using a multiple lysogen where, unlike the multiple lysogens studied here where both phages were inducible by the activated SOS system, in the Bertani experiment one phage was inducible by the SOS system, while the other was not. Examination of phage spontaneously released from single bacterium of the latter type of multiple lysogen, shows that spontaneous induction of the two types of phages are independent events, a single bacterial lysogen even though carrying both prophage types spontaneously produces one or the other.

\section{On the differences in spontaneous induction of $H-19 B$ and $\lambda$}

The results with the mutant $\lambda$ prophages allowed us to rule out one possible explanation for the lower spontaneous induction of the $\lambda$ prophage compared to induction of $\mathrm{H}-19 \mathrm{~B}$, namely, that in addition to activated RecA another bacterial factor(s) contributes to the induction of $\mathrm{H}-19 \mathrm{~B}$ but not $\lambda$. The observation that the $\lambda c / 857$ ind $^{+}$and $\lambda \mathrm{O}_{\mathrm{R}} 323$ prophage cause a significant reduction in CamR colonies in SIVET IIT shows that both these $\lambda$ derivative prophages are efficiently induced in the same cells in which the $\mathrm{H}$ 19B prophage is induced. Because the repressor encoded by $\mathrm{O}_{\mathrm{R}} 323$ and the operator sites encoded by c/857 ind ${ }^{+}$are identical to those encoded by the wild-type $\lambda$, the difference in the induction of $\mathrm{H}-19 \mathrm{~B}$ and $\lambda$ measured by the SIVET IIT test can not reflect a difference in repressor response to a host factor and therefore must reflect differences in repressor action with the operator regions of the two phages. Thus, spontaneous induction of prophages with $\lambda$ immunity results from a response to the same environmental signal(s) that acts to spontaneously induce the $\mathrm{H}-19 \mathrm{~B}$ prophage.

\section{Prophage inducibility and Stx production}

One striking finding from these studies is that the two phages that spontaneously induce most readily, or by the terminology of Little et al. (1999) have 'hair triggers', are those with stx genes, $\mathrm{H}-19 \mathrm{~B}$ and $933 \mathrm{~W}$. Those authors proposed that the stability of the repressor-operator system of $\lambda$ and presumably those of other lambdoid phages have evolved so that prophage induction occurs only when the health of the host bacterium is significantly compromised. They argue that an increased sensitivity to inducing stimuli may decrease the relative fitness of the $\lambda$ prophage by causing it to induce even under conditions that are favourable to the survival of the host bacterium and its prophage. Whereas this reasoning may be applicable to prophages not encoding Stx, it is unlikely to apply to $\mathrm{H}-19 \mathrm{~B}$ and $933 \mathrm{~W}$. If, as we have previously proposed (Neely and Friedman, 1998a; Wagner et al., 2001b; Wagner et al., 2002), induction of Stx-producing prophages plays a significant role in the expression and release of Stx, these Stx encoding prophages may be subject to selective pressures not operating on other prophages. The assumption underlying this argument is that both modes of a temperate phage existence have an impact on temperate phage evolution; if the phage in its prophage state contributes to the growth advantage of its bacterial host, the responsible trait(s) and the mechanism controlling its expression, in this case a higher inducibility, will be selected even though the selected trait may not provide a selective advantage to the phage during lytic growth. Assuming that the small fraction of the STEC lysogens that is spontaneously induced contributes in a significant way to Stx expression and/or release, then an increase in the induced fraction could significantly influence the level of Stx production and/or release. If Stx contributes to the fitness of the STEC strain to its environment, then it seems plausible that there could be selective pressure on the prophage to induce more readily, explaining why, of phages we examined, those that exhibit substantially greater spontaneous induction are the Stx encoding phages $\mathrm{H}-19 \mathrm{~B}$ and $933 \mathrm{~W}$. But how can selection at the level of the lysogen work if, by this argument, the very lysogens that are responsible for Stx production and/or release are those lysogens that are destroyed by the induced prophage? Although being high relative to lysogens with prophages not carrying stx genes, the fraction of spontaneously induced lysogens with prophages carrying stx genes is still low; most of the lysogens survive. Therefore, assuming that Stx provides a growth advantage to the STEC strain, we suggest that, based on what admittedly is a small sample, the selection is for the remaining population of lysogens of the clone from which the destroyed lysogens were derived. A few have been sacrificed to give a growth advantage to the many. The desired trait expressed by the dead lysogens is maintained in the population of survivors; the diarrhoea caused by Stx promotes the spread of the surviving descendents of the original clone. 


\section{Experimental procedures}

\section{Bacteria, phage, and plasmids}

Bacterial strains, relevant genotypes, and sources are listed in Table 1. Phages $\lambda, \lambda c / 857$ ind $^{+}, \lambda i m m 434$, and P21 (21GP) were obtained from the $\mathrm{NIH}$ collection. Phage $\lambda \mathrm{O}_{\mathrm{R}} 323$ was isolated from strains JL5938 provided by J. Little. Phages $\mathrm{H}$ $19 \mathrm{~B}$ and $933 \mathrm{~W}$ were isolated from strains $\mathrm{C} 600(\mathrm{H}-19 \mathrm{~B})$ and $\mathrm{C600}(933 \mathrm{~W})$, respectively, which were provided by $A$. O'Brien.

To construct pJLTnpR, the tnpR gene with its associated RBS was PCR amplified from pIVET5 (see below) with primers 39 and 40 (Supplementary material, Table S1) and cloned into the expression vector pTrc99A (Amersham Biosciences) as described (Sambrook and Fritsch, 1989).

\section{Sources of resistance markers, tnpR and res sites}

The tet and amp genes were amplified from vector pBR322 (Sutcliffe, 1979). The cat and kan genes were amplified from plasmids pKan (Carolina Biological Supply Company) and pBR325 (Bolivar, 1978) respectively. The cat-sacB cassette was amplified from strain DY406. The res sites and tnpR gene were amplified from vectors pAC21 and pIVET5 (Camilli and Mekalanos, 1995) respectively.

\section{Media}

LB and top agar media were prepared as described (Miller and Friedman, 1980). Except where noted, chloramphenicol, kanamycin, ampicillin, tetracycline, and mitomycin C (Sigma) were used at final concentrations of $10 \mu \mathrm{g} \mathrm{ml}^{-1}, 20 \mu \mathrm{g} \mathrm{ml}^{-1}$, $30 \mu \mathrm{g} \mathrm{ml}^{-1}, 5 \mu \mathrm{g} \mathrm{ml}^{-1}$ and $2 \mu \mathrm{g} \mathrm{ml}^{-1}$ respectively. The selective media used in obtaining sucrose resistant derivatives was LB media without $\mathrm{NaCl}$ supplemented with $20 \%$ sucrose (Sigma) and $50 \mu \mathrm{g} \mathrm{ml}^{-1}$ kanamycin.

\section{General strain construction methods}

Relevant strains are listed in Table 1. Except where indicated, all strain constructions utilized the $\lambda$ Red based recombination system (Court et al., 2002; Thomason et al., 2003). The following is a brief outline of the procedure. Sequences to be recombined were amplified by PCR with primers that included on their $5^{\prime}$ ends $35-40 \mathrm{bp}$ of homology to the regions flanking the target insertion site. The sequences of primers used in strain construction are listed in Supplementary material, Table S1. Following column purification (Quaigen), these amplicons were electroporated into derivatives of strain DY378, which carries a defective $\lambda$ prophage. This prophage encodes the c/857 ts repressor allele and therefore expresses the lambda recombination functions at high temperature. Transient expression of these recombina-

Table 1. Bacterial strains.

\begin{tabular}{|c|c|c|}
\hline Strain & Relevant genotype & Source \\
\hline DY378 & See Experimental procedures & D. Court \\
\hline DY406 & W3110 ( $\lambda$ c/857 N-kil::cat-sacB $)$ & D. Court \\
\hline K7523 & $\mathrm{C} 600(\mathrm{H}-19 \mathrm{~B})$ & A. O'Brien \\
\hline K8443 & C600(933 W) & A. O'Brien \\
\hline K9723 & DY378(H-19B $\Delta N:: k a n)$ & This laboratory \\
\hline K9725 & DY378(H-19B) & This laboratory \\
\hline K9834 & MC1000(H-19B) & This laboratory \\
\hline K9979 & K9981 galK::res & This laboratory \\
\hline K9981 & DY378(H-19B) pKan & This laboratory \\
\hline K9988 & DY378(H-19B $\Delta N:: k a n \Delta O P:: a m p)$ & This laboratory \\
\hline K9989 & K9981 galK::res-tet-res pKan & This laboratory \\
\hline K10045 & MC1000(mult H-19B) galK::res-tet-res & This laboratory \\
\hline K10373 & DY378 galK::cat & This laboratory \\
\hline K10376 & DY378 galK::resC-tet-resC & This laboratory \\
\hline K10447 & MC1000(mult H-19B) galK::resC-tet-resC & This laboratory \\
\hline K10448 & MC1000(H-19B $\Delta N:: k a n$ cro-tnpR $\Delta O P:: a m p)$ galK::resC-tet-resC & This laboratory \\
\hline K10449 & MC1000(H-19B $\Delta N:: k a n$ cro-tnpR168 $\Delta O P:: a m p)$ galK::resC-tet-resC & This laboratory \\
\hline K10450 & MC1000(H-19B $\Delta N:: k a n$ cro-tnpR184 $\Delta O P:: a m p)$ galK::resC-tet-resC & This laboratory \\
\hline K10484 & 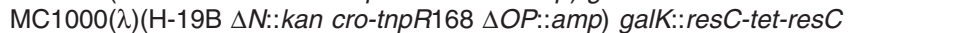 & This laboratory \\
\hline K10487 & MC1000(H-19B $\Delta N:: k a n$ cl-ind cro-tnpR $\Delta O P:: a m p)$ galK::resC-tet-resC & This laboratory \\
\hline K10488 & MC1000(H-19B $\Delta N:: k a n$ cl-ind cro-tnpR168 $\Delta O P:: a m p)$ galK::resC-tet-resC & This laboratory \\
\hline K10490 & MC1000(H-19B)(H-19B $\Delta N:: k a n$ cro-tnpR $\Delta O P:: a m p)$ galK::resC-tet-resC & This laboratory \\
\hline K10491 & MC1000(H-19B)(H-19B $\Delta N:: k a n$ cro-tnpR168 $\Delta O P:: a m p)$ galK::resC-tet-resC & This laboratory \\
\hline K10492 & MC1000(H-19B) $(\mathrm{H}-19 \mathrm{~B} \Delta N:: k a n$ cro-tnpR184 $\Delta O P:: a m p)$ galK::resC-tet-resC & This laboratory \\
\hline K10493 & DY378(H-19B $\Delta N:: k a n$ cl:::cat-sacB cro-tnpR184 $\Delta O P:: a m p)$ & This laboratory \\
\hline K10494 & DY378(H-19B $\Delta N:: k a n$ cl-ind cro- cat-sacB $\Delta O P:: a m p)$ & This laboratory \\
\hline K10556 & MC1000 $\left(\lambda c / 857\right.$ Ind $\left.^{+}\right)(\mathrm{H}-19 \mathrm{~B} \Delta N::$ kan cro-tnpR168 $\Delta O P:: a m p)$ galK::resC-tet-resC & This laboratory \\
\hline K10559 & MC1000(933 W)(H-19B $\Delta N:: k a n$ cro-tnpR168 $\Delta O P:: a m p)$ galK::resC-tet-resC & This laboratory \\
\hline K10579 & MC1000(933 W $\Delta N::$ kan cro-tnpR168 $\Delta O P:: a m p)$ galK::resC-tet-resC & This laboratory \\
\hline K10583 & MC1000(P21)(H-19B $\Delta N:: k a n$ cro-tnpR168 $\Delta O P:: a m p)$ galK:: resC-tet-resC & This laboratory \\
\hline K10637 & MC1000 galK:: res-tet-res & This laboratory \\
\hline K10638 & MC1000 $(\lambda i m m 434)(\mathrm{H}-19 \mathrm{~B} \Delta N:: k a n$ cro-tnpR168 $\Delta O P:: a m p)$ galK::resC-tet-resC & This laboratory \\
\hline K10640 & MC1000 $\left(\lambda \mathrm{O}_{\mathrm{B}} 323\right)(\mathrm{H}-19 \mathrm{~B} \Delta N:: k a n$ cro-tnpR168 $\Delta O P:: a m p)$ galK::resC-tet-resC & This laboratory \\
\hline
\end{tabular}


tion factors shortly before transformation allows for efficient recombination between the linear fragment and target sequences. Recombinants with a transferred antibiotic resistance gene were selected by plating transformed cultures on LB agar plates supplemented with antibiotics at concentrations noted above. Recombinants in which the amplicon had replaced the cat-sacB cassette were selected on sucrose media then screened for sensitivity to chloramphenicol. Insertion of the amplicon in the proper location was confirmed by PCR using one insert-specific primer and one primer specific to sequence outside the site of insertion.

Table S1 listing sequences of all primers is found in the Supplementary material.

\section{Construction of galK::cat::resC-tet-resC}

In constructing galK::cat::resC-tet-resC, the chromosomal galK gene of strain K9981 (DY378-H-19B-pKan) was replaced by the cat-sacB cassette, which subsequently was replaced with the resC site. Subsequent steps in construction are elaborated in Fig. 8. The general strategy of using recombination rather than PCR amplification to create the resC-tetres $C$ construct was adopted to avoid the problems inherent in PCR amplification of direct repeats, such as primer dimers and the amplification of one of the res $C$ sites rather then the complete amplicon resulting from hybridization of both resCspecifc primers to the same direct repeat. $\mathrm{P} 1$ transduction of galK::cat::resC-tet-resC to strain K9834 yielded strains K10447.

\section{Construction of the SIVET lysogens and SIVET lysogen derivatives carrying a second, intact $\mathrm{H}-19 \mathrm{~B}$ prophage}

The essential features of the SIVET prophage are pictured in Fig. 2. The $N$ gene of the $\mathrm{H}-19 \mathrm{~B}$ prophage of strain K9725 was replaced with a kan gene yielding strain K9723. The $\mathrm{H}-$ 19B OP genes of K9723 were replaced with an amp gene yielding strain K9988. Insertion of the tnp $R$ gene required two recombination events. First, the cat-sacB cassette was placed downstream of the cro gene of the $\mathrm{H}-19 \mathrm{~B}$ prophage in K9988. Replacement of the cat-sacB cassette by the wildtype tnpR, tnpR168, or tnpR184 genes yielded a prophage with the tnpR gene downstream of the $P_{\mathrm{R}}$ promoter. The specific nucleotide changes carried in the RBS of tnpR168 and tnpR184 have been previously reported (Lee et al., 1999). The $\mathrm{H}-19 \mathrm{~B}$ prophages with $\Delta N:: k a n, \Delta O P:: a m p$ and cro-tnpR constitute the SIVET $\mathrm{H}-19 \mathrm{~B}$ prophages. $\mathrm{P} 1$ transduction of the SIVET ${ }^{\mathrm{WT}}$ prophage to strain K10045 (MC1000 galK::res-tet-res) yielded strain K10637. The SIVET ${ }^{\mathrm{WT}}$, SIVET ${ }^{168}$, and SIVET ${ }^{184}$ prophages were also $\mathrm{P} 1$ transduced to strain K10447 (MC1000 galk::cat::resC-tet-resC). Strain K10447 carries multiple tandem copies of the wild type $\mathrm{H}$ 19B prophage. This transduction of the SIVET prophages yielded two distinct classes of lysogens. Strains K10448, K10449, K10450, in which the SIVET prophage had replaced all of the wt $\mathrm{H}-19 \mathrm{~B}$ prophages, carry only a single SIVET prophage. Strains K10490, K10491, and K10492, in which recombination of the SIVET Iysogen had failed to remove all tandem copies of the wild-type prophage carry both a SIVET prophage and at least one wild-type $\mathrm{H}$-19B prophage. These
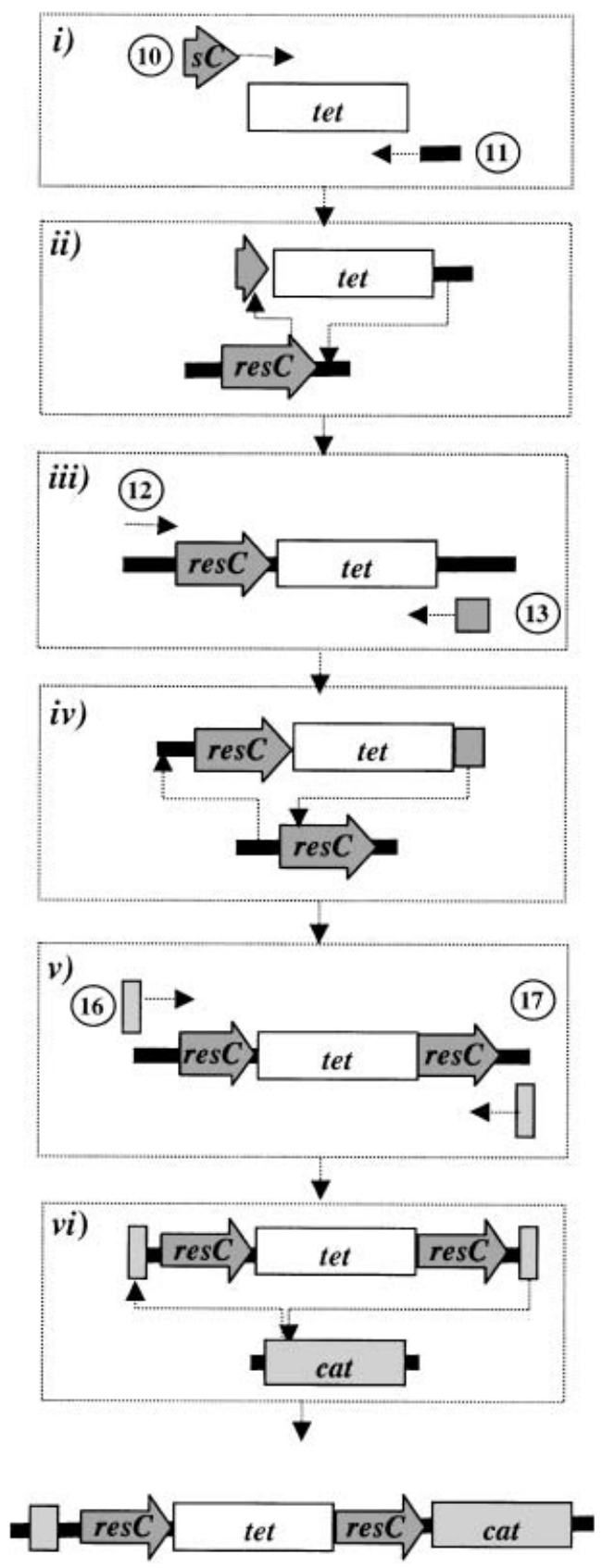

Fig. 8. Construction of galK::cat::resC-tet-resC. Primer sequences can be found in Table S1 in Supplementary Material (i) Primers 10 and 11 were used to fuse res $C$ and galK sequences, respectively, to $t$ he $t^{R} t^{R}$ gene. (ii) The tet gene was recombined directly $3^{\prime}$ to the chromosomally inserted res $C$ site. (iii) The resC-tet insertion was amplified using primers 12 and 13 . (iv) The resulting amplicon was recombined $5^{\prime}$ of galk::resC yielding strain K10376. (v) The resC-tetres $C$ construct was amplified from strain $\mathrm{K} 10376$ using primers 16 and 17 and (vi) recombined between the sixth and seventh codon of the chromosomally inserted cat gene in strain K10373 to produce galK::cat::resC-tet-resC.

two groups were distinguished using PCR primers flanking the site of tnpR insertion, with SIVET lysogens giving one band and SIVET(wt-H-19B) lysogens producing a second, smaller band. 
To construct the $\mathrm{H}-19 \mathrm{~B}$ clind SIVET prophages, the conserved serine codon residue in the $\mathrm{cl}$ gene identified as potentially required for autocleavage (J.W. Little, pers. comm.) was replaced by the cat-sacB cassette in the SIVET ${ }^{184}$ prophage to produce strain K10493. The cat-sacB cassette was then replaced with a ssDNA oligo encoding an alanine codon at the position of the conserved serine codon flanked by 36 and 42 bp of $c /$ homology, respectively, on each side. This yielded strain K10494. The $c /$ genes of two of these recombinants were PCR amplified and sequenced to confirm the presence of the designed mutation and to insure the absence of secondary mutations. To construct the clind derivatives of the SIVET ${ }^{\mathrm{WT}}$ and SIVET ${ }^{168}$ prophages, the tnpR184 gene in the prophage in strain K10494 was replaced by the cat-sacB cassette, which was subsequently replaced

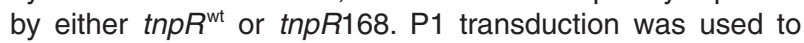
move the clind SIVET prophages to strain K10447, yielding the SIVET strains K10487, K10488 and K10489 with noninducible prophages.

\section{Construction of the 933 W SIVET ${ }^{168}$}

The $N$ gene of the $933 \mathrm{~W}$ prophage in strain $\mathrm{K} 9857$ was replaced with kan. Replacement of the $933 \mathrm{~W} O P$ genes with amp and insertion of the tnpR gene was achieved in two steps. First, the cat-sacB cassette was placed downstream of the cro gene in the $933 \mathrm{~W} \Delta \mathrm{N}:$ :kan prophage yielding the $933 \mathrm{~W} \Delta N$ :: kan cro-cat-sacB prophage. Second, primers with homology to the $933 \mathrm{~W}$ cro gene and to sequence downstream of the $933 \mathrm{~W} P$ gene, respectively, were used to amplify the tnpR gene and adjoining $\triangle O P:$ :amp sequence from SIVET ${ }^{168}$. This amplicon was used to replace cat-sacB and the $O$ and $P$ genes in the $933 \mathrm{~W} \Delta N$ ::kan cro-cat-sacB prophage, yielding the 933 W SIVET ${ }^{168}$ prophage. This prophage was transduced using P1 into strain K10636 to yield the 933 W SIVET lysogen.

\section{Construction of SIVET ${ }^{168}$ lysogens carrying a second intact prophage other than $\mathrm{H}-19 B$}

Top agar $(2.5 \mathrm{ml})$ containing approximately $50 \mu \mathrm{l}$ of SIVET ${ }^{168}$ overnight culture was overlaid on LB + tetracycline plates. One hundred microlitres of the appropriate phage lysate was spotted on the bacterial lawn and the plate was incubated overnight at $37^{\circ} \mathrm{C}\left(32^{\circ} \mathrm{C}\right.$ for $\left.\lambda c / 857 i d^{+}\right)$. Bacteria from the zone of lysis were struck out on LB + tetracycline and grown overnight. Colonies were tested for the second phage by determining if they were immune to infection by a clear derivative of the second phage, but sensitive to a phage with the same adsorption specificity but a different immunity (Gottesman and Yarmolinsky, 1968).

\section{Measuring the frequency of SIVET induction}

To measure spontaneous induction of SIVET lysogens, overnight cultures grown in LB supplemented with tetracycline, kanamycin and ampicillin were diluted 1:100 in LB and grown overnight at 32 or $37^{\circ} \mathrm{C}$. Dilutions of these cultures were transferred to LB agar plates supplemented with either kanamycin and ampicillin (to select for all SIVET lysogens) or kanamycin, ampicillin, and chloramphenicol (to select for induced SIVET lysogens). Plates were incubated at 32 or $37^{\circ} \mathrm{C}$ for $48 \mathrm{~h}$. To measure induction or survival following exposure to mitomycin $\mathrm{C}$, one $\mathrm{ml}$ cultures of LB inoculated with $10 \mu \mathrm{l}$ of overnight culture were grown to exponential phase, diluted $1: 2$ in LB supplemented with mitomycin C and grown for an additional 2-4 $\mathrm{h}$. The bacteria were then pelleted, washed twice in LB, resuspended in $4 \mathrm{ml} \mathrm{LB}$, grown for $4 \mathrm{~h}$ at $37^{\circ} \mathrm{C}$, and plated at appropriate dilutions on selective plates as described above.

\section{Acknowledgements}

The authors thank John Little for help in identifying codons to be changed in the construction of the ind mutant. Victor DiRita and Melissa Mills are thanked for helpful comments on the manuscript. Andrew Camilli and Sankar Adhya are thanked for bacterial strains and phage. James Bardwell is thanked for helpful suggestions. Richard Calendar is thanked for pointing out the Bertani finding. This work was supported by Public Health Grant Al11459-10. J.L. was supported in part by NIH Training Grants GM007315 and AI07528.

\section{Supplementary material}

The following material is available from http://www.blackwellpublishing.com/products/journals/ suppmat $/ \mathrm{mmi} / \mathrm{mmi} 3934 / \mathrm{mmi} 3934 \mathrm{sm} . \mathrm{htm}$

Table S1. Sequences and reference numbers of primers used for strain construction.

\section{References}

Barksdale, L., and Arden, S.B. (1974) Persisting bacteriophage infections, lysogeny, and phage conversions. Annu Rev Microbiol 28: 265-299.

Bertani, G. (1951) Studies of Lysogenesis. I. The mode of phage liberation by lysogenic Escherichia coli. J Bacteriol 62: 293-300.

Bochner, B.R., Huang, H.C., Schieven, G.L., and Ames, B.N. (1980) Positive selection for loss of tetracycline resistance. $J$ Bacteriol 143: 926-933.

Bolivar, F. (1978) Construction and characterization of new cloning vehicles. III. Derivatives of plasmid pBR322 carrying unique Eco RI sites for selection of Eco RI generated recombinant DNA molecules. Gene 4: 121-136.

Boyd, E.F., and Brussow, H. (2002) Common themes among bacteriophage-encoded virulence factors and diversity among the bacteriophages involved. Trends Microbiol 10: 521-529.

Brooks, K., and Clark, A.J. (1967) Behavior of lambda bacteriophage in a recombination deficienct strain of Escherichia coli. J Virol 1: 283-293.

Broudy, T.B., Pancholi, V., and Fischetti, V.A. (2001) Induction of lysogenic bacteriophage and phage-associated toxin from group a streptococci during coculture with human pharyngeal cells. Infect Immun 69: 14401443. 
Calderwood, S.B., Auclair, F., Donohue-Rolfe, A., Keusch, G.T., and Mekalanos, J.J. (1987) Nucleotide sequence of the Shiga-like toxin genes of Escherichia coli. Proc Natl Acad Sci USA 84: 4364-4368.

Camilli, A., and Mekalanos, J.J. (1995) Use of recombinase gene fusions to identify Vibrio cholerae genes induced during infection. Mol Microbiol 18: 671-683.

Camilli, A., Beattie, D.T., and Mekalanos, J.J. (1994) Use of genetic recombination as a reporter of gene expression. Proc Natl Acad Sci USA 91: 2634-2638.

Court, D.L., and Oppenheim, A. (1983) Phage lambda's accessory genes. In Lambda II. Hendrix, R.W., Roberts, J.W., Stahl, F.W., and Weisberg, R.A., (eds). Cold Spring Harbor, NY: Cold Spring Harbor Laboratory, pp. 251-278.

Court, D.L., Sawitzke, J.A., and Thomason, L.C. (2002) Genetic engineering using homologous recombination. Annu Rev Genet 36: 361-388.

Datz, M., Janetzki-Mittmann, C., Franke, S., Gunzer, F., Schmidt, H., and Karch, H. (1996) Analysis of the enterohemorrhagic Escherichia coli O157 DNA region containing lambdoid phage gene $\mathrm{P}$ and Shiga-like toxin structural genes. Appl Environ Microbiol 62: 791-797.

De Grandis, S., Ginsberg, J., Toone, M., Climie, S., Friesen, J., and Brunton, J. (1987) Nucleotide sequence and promoter mapping of the Escherichia coli Shiga-like toxin operon of bacteriophage $\mathrm{H}-19 \mathrm{~B}$. J Bacteriol 169: 43134319.

Dodd, I.B., Perkins, A.J., Tsemitsidis, D., and Egan, J.B. (2001) Octamerization of lambda $\mathrm{Cl}$ repressor is needed for effective repression of $\mathrm{P}(\mathrm{RM})$ and efficient switching from lysogeny. Genes Dev 15: 3013-3022.

Eisen, H., Pereira da Silva, L., and Jacob, F. (1968) The regulation and mechanism of DNA synthesis in bacteriophage lambda. Cold Spring Harb Symp Quant Biol 33: 755764.

Friedberg, E.C., Walker, G.C., and Siede, W.S. (1995) DNA repair and mutagenesis. Washington, DC: Am Soc Microbiol Press.

Fuchs, S., Muhldorfer, I., Donohue-Rolfe, A., Kerenyi, M., Emody, L., Alexiev, R., et al. (1999) Influence of RecA on in vivo virulence and Shiga toxin 2 production in Escherichia coli pathogens. Microb Pathog 27: 13-23.

Gottesman, M.E., and Yarmolinsky, M.B. (1968) Integrationnegative mutants of bacteriophage lambda. J Mol Biol 31: 487-505.

Grindley, N.D. (1983) Transposition of Tn3 and related transposons. Cell 32: 3-5.

Gussin, G.N., Johnson, A.D., Pabo, C.O., and Sauer, R.T. (1983) Repressor and Cro protein: structure, function, and role in lysogenization. In Lambda II. Hendrix, R.W., Roberts, J.W., Stahl, F.W., and Weisberg, R.A., (eds). Cold Spring Harbor, NY: Cold Spring Harbor Laboratory, pp. 93-121.

Heffron, F. (1983) Tn3 and its relatives. In Mobile Genetic Elements. Shapiro, J.A., (ed). New York, NY: Academic Press, pp. 223-260.

Hendrix, R.W., Roberts, J.W., Stahl, F.W., and Weisberg, R.A. (1983) Lambda II. Cold Spring Harbor, NY: Cold Spring Harbor Laboratory.

Hertman, I., and Luria, S.E. (1967) Transduction studies on the role of a rec+ gene in the ultraviolet induction of prophage lambda. J Mol Biol 23: 117-133.
Heyderman, R.S., Soriani, M., and Hirst, T.R. (2001) Is immune cell activation the missing link in the pathogenesis of post-diarrhoeal HUS? Trends Microbiol 9: 262-266.

Karch, H., Bielaszewska, M., Bitzan, M., and Schmidt, H. (1999) Epidemiology and diagnosis of Shiga toxin-producing Escherichia coli infections. Diagn Microbiol Infect Dis 34: 229-243.

Kim, B., and Little, J.W. (1993) LexA and lambda Cl repressors as enzymes: specific cleavage in an intermolecular reaction. Cell 73: 1165-1173.

Lee, S.H., Hava, D.L., Waldor, M.K., and Camilli, A. (1999) Regulation and temporal expression patterns of Vibrio cholerae virulence genes during infection. Cell 99: 625634.

Levine, A., Bailone, A., and Devoret, R. (1979) Cellular levels of the prophage lambda and 434 repressors. $J \mathrm{Mol}$ Biol 131: 655-661.

Lin, L.L., and Little, J.W. (1988) Isolation and characterization of noncleavable (Ind-) mutants of the LexA repressor of Escherichia coli K-12. J Bacteriol 170: 2163-2173.

Little, J.W. (1984) Autodigestion of lexA and phage lambda repressors. Proc Natl Acad Sci USA 81: 1375-1379.

Little, J.W., and Mount, D.W. (1982) The SOS regulatory system of Escherichia coli. Cell 29: 11-22.

Little, J.W., Shepley, D.P., and Wert, D.W. (1999) Robustness of a gene regulatory circuit. EMBO J 18: 4299-4307.

Lwoff, A. (1953) Lysogeny. Bacteriol Rev 17: 269-337.

Miller, H.I., and Friedman, D.I. (1980) An E. coli gene product required for lambda site-specific recombination. Cell 20: 711-719.

Neely, M.N., and Friedman, D.I. (1998a) Functional and genetic analysis of regulatory regions of coliphage $\mathrm{H}-19 \mathrm{~B}$ : location of shiga-like toxin and lysis genes suggest a role for phage functions in toxin release. Mol Microbiol 28: 1255-1267.

Neely, M.N., and Friedman, D.I. (1998b) Arrangement and functional identification of genes in the regulatory region of lambdoid phage $\mathrm{H}-19 \mathrm{~B}$, a carrier of a Shiga-like toxin. Gene 223: 105-113.

Newland, J.W., and Neill, R.J. (1988) DNA probes for Shigalike toxins I and II and for toxin-converting bacteriophages. J Clin Microbiol 26: 1292-1297.

O'Brien, A.D., Newland, J.W., Miller, S.F., Holmes, R.K., Smith, H.W., and Formal, S.B. (1984) Shiga-like toxinconverting phages from Escherichia coli strains that cause hemorrhagic colitis or infantile diarrhea. Science 226: 694696.

Pirrotta, V., and Ptashne, M. (1969) Isolation of the 434 phage repressor. Nature 222: 541-544.

Plunkett, G., 3rd, Rose, D.J., Durfee, T.J., and Blattner, F.R. (1999) Sequence of Shiga toxin 2 phage 933W from Escherichia coli O157: H7: Shiga toxin as a phage lategene product. J Bacteriol 181: 1767-1778.

Ptashne, M. (1992) A Genetic Switch. Cambridge, MA: Cell Press and Blackwell Publications.

Reed, R.R., Shibuya, G.I., and Steitz, J.A. (1982) Nucleotide sequence of gamma delta resolvase gene and demonstration that its gene product acts as a repressor of transcription. Nature 300: 381-383.

Revet, B., von Wilcken-Bergmann, B., Bessert, H., Barker, A., and Muller-Hill, B. (1999) Four dimers of lambda repres- 
sor bound to two suitably spaced pairs of lambda operators form octamers and DNA loops over large distances. Curr Biol 9: 151-154.

Roberts, J.W., and Devoret, R. (1983) Lysogenic induction. In Lambda II. Hendrix, R.W., Roberts, J.W., Stahl, F.W., and Weisberg, R.A., (eds). Cold Spring Harbor, NY: Cold Spring Harbor Laboratory, pp. 123-144.

Sambrook, J., and Fritsch, E.M. (1989) Molecular Cloning, A laboratory Manual. Cold Spring Harbor, NY: Cold Spring Harbor Press.

Shi, T., and Friedman, D.I. (2001) The operator-early promoter regions of Shiga-toxin bearing phage $\mathrm{H}-19 \mathrm{~B}$. Mol Microbiol 41: 585-599.

Shinagawa, H., and Ito, T. (1973) Inactivation of DNA-binding activity of repressor in extracts of lambda-lysogen treated with mitomycin C. Mol Gen Genet 126: 103-110.

Sjogren, R., Neill, R., Rachmilewitz, D., Fritz, D., Newland, J., Sharpnack, D., et al. (1994) Role of Shiga-like toxin I in bacterial enteritis: comparison between isogenic Escherichia coli strains induced in rabbits. Gastroenterology 106: 306-317.

Smith, H.W., and Linggood, M.A. (1971) The transmissible nature of enterotoxin production in a human enteropathogenic strain of Escherichia coli. J Med Microbiol 4: 301305.

Sussman, R., and Jacob, F. (1962) Sur un systeme de repression thermosensible ch2 le bacteriophage 1 d'Escherichia coli. C R Acad Sci Paris 254: 1517-1519.

Sutcliffe, J.G. (1979) Complete nucleotide sequence of the Escherichia coli plasmid pBR322. Cold Spring Harb Symp Quant Biol 43 Part 1: 77-90.
Thomason, L.C., Bubunenko, M., Constantino, N., Wilson, H.R., Oppenheim, A., and Court, D.L. (2003) Recombineering-genetic engineering in bacteria using homologous recombination. In Current Protocols in Molecular Biology. Ausubel, F.M., Brent, R.E., Kingston, R.E., Moore, D.D., Seidman, J.G., Smith, K., and Struhl, K., (eds). John Wiley and Sons, Unit.1.16.

Toman, Z., Dambly-Chaudiere, C., Tenenbaum, L., and Radman, M. (1985) A system for detection of genetic and epigenetic alterations in Escherichia coli induced by DNAdamaging agents. J Mol Biol 186: 97-105.

Unkmeir, A., and Schmidt, H. (2000) Structural analysis of phage-borne stx genes and their flanking sequences in shiga toxin-producing Escherichia coli and Shigella dysenteriae type 1 strains. Infect Immun 68: 48564864.

Wagner, P.L., and Waldor, M.K. (2002) Bacteriophage control of bacterial virulence. Infect Immun 70: 3985-3993.

Wagner, P.L., Acheson, D.W., and Waldor, M.K. (2001a) Human neutrophils and their products induce Shiga toxin production by enterohemorrhagic Escherichia coli. Infect Immun 69: 1934-1937.

Wagner, P.L., Neely, M.N., Zhang, X., Acheson, D.W., Waldor, M.K., and Friedman, D.I. (2001b) Role for a phage promoter in Shiga toxin 2 expression from a pathogenic Escherichia coli strain. J Bacteriol 183: 2081-2085.

Wagner, P.L., Livny, J., Neely, M.N., Acheson, D.W., Friedman, D.I., and Waldor, M.K. (2002) Bacteriophage control of Shiga toxin 1 production and release by Escherichia coli. Mol Microbiol 44: 957-970. 